\title{
Constitutive Suppression of PRL-3 Inhibits Invasion and Proliferation of Gastric Cancer Cell in vitro and in vivo
}

\author{
Yasuko Matsukawa ${ }^{a}$ Shuho Semba ${ }^{a}$ Hirotaka Kato $^{a}$ Yu-ichiro Koma ${ }^{a}$ \\ Kazuyoshi Yanagiharab Hiroshi Yokozaki ${ }^{a}$ \\ a Division of Pathology, Department of Pathology, Kobe University Graduate School of Medicine, Kobe, and \\ ${ }^{b}$ Laboratory of Health Sciences, Department of Life Sciences, Yasuda Women's University Faculty of Pharmacology, \\ Hiroshima, Japan
}

\section{Key Words}

Phosphatase of regenerating liver-3 - Gastric cancer •

Small interfering ribonucleic acid $\cdot$ Invasion $\cdot$ Proliferation of metastasis but also to the growth of primary foci of human gastric cancer. Therefore, PRL-3 may be one of the target molecules in gastric cancer therapy.

Copyright ๑ 2010 S. Karger AG, Basel

\begin{abstract}
Objective: Overexpression of phosphatase of regenerating liver-3 (PRL-3) has been implicated in tumor progression and metastasis of gastric carcinoma. Here we examined what alterations occur in the phenotype of gastric cancer cells in vitro and in vivo when PRL-3 expression is knocked down. Methods: We constructed a small interfering RNA (siRNA)expressing vector which stably interfered with PRL-3 expression and was transfected into SH101-P4 cells, which express the highest $P R L-3$ mRNA levels among 13 gastric cancer cell lines. The new SH101-P4 subclones, in which PRL-3 was stably reduced, were established and their in vitro growth, motility and abilities of liver metastasis from the injected spleen were analyzed in vivo. Results: PRL-3 knockdown effectively suppressed invasion and growth of SH101-P4 cells in vitro. Liver metastasis in vivo was significantly decreased when PRL-3 expression was suppressed. The primary tumor size in the injected spleen tended to be smaller in PRL-3 knockdown clones than in the controls. These findings suggest that PRL3 expression may contribute not only to the establishment
\end{abstract}

\section{Introduction}

Although gastric cancer has gradually decreased in prevalence, it still accounts for a large portion of cancerrelated deaths in Japan. Like other cancers, growth and metastatic abilities are the main lethal processes which determine its malignancy. Protein tyrosine phosphatases play a fundamental role in regulating diverse proteins that participate essentially in every aspect of cellular physiology and pathogenic processes [1]. Phosphatase of regenerating liver- $1,-2$ and -3 (PRL-1, -2 and -3 ) represent a novel class of the superfamily of protein tyrosine phosphatases that are ubiquitously expressed in various organs and are up-regulated in the regenerating liver $[2,3]$. Interestingly, overexpression of PRL-1 and PRL-2 has been found to transform mouse fibroblasts and hamster pancreatic epithelial cells in culture and to promote tumor growth in nude mice $[2,3]$. Similarly, PRL-3 enhances the growth of human embryonic kidney fibroblasts [4].

\section{KARGER}

Fax +41613061234 E-Mail karger@karger.ch www.karger.com
(ㄷ) 2010 S. Karger AG, Basel

$1015-2008 / 10 / 0773-0155 \$ 26.00 / 0$

Accessible online at:

www.karger.com/pat
Dr. Hiroshi Yokozaki

Division of Pathology, Department of Pathology

Kobe University Graduate School of Medicine

7-5-1 Kusunoki-cho, Chuo-ku, Kobe 650-0017 (Japan)

Tel. +81 78382 5460, Fax +81 78382 5479, E-Mail hyoko@med.kobe-u.ac.jp 
In addition to growth ability, overexpression of PRL-3 in Chinese hamster ovary cells promotes in vitro cell motility and invasion [5]. Several other reports have been published, suggesting the involvement of PRL-3 in cell motility not only in experimental in vitro and in vivo models but also in clinical human cancers, such as malignant melanoma, breast cancer, pancreatic cancer, ovarian cancer and nasopharyngeal cancer [6-11].

Previously, we have examined gastric and colon cancer tissues and found that higher levels of $P R L-3$ mRNA were correlated with distant metastases [12, 13]. Moreover, in a colon cancer model, we showed an effect on in vivo metastasis by using the small interfering RNA (siRNA) method by which $P R L-3$ mRNA expression was transiently suppressed [13]. Since there were only few reports on the role of PRL-3 in gastric cancer metastasis in vitro and in vivo, we decided to conduct this study using gastric cancer cells.

In this report, to evaluate the long-term effect of inhibition of PRL-3 expression on cancer cells, we constructed new subclones of the SH101-P4 gastric cancer cell line, in which $P R L-3$ expression was stably reduced by transfecting the siRNA PRL-3 vector. Using PRL-3 knockdown cells, we examined how PRL-3 contributed to cell proliferation and invasion ability in vitro. Furthermore, the role of PRL-3 was investigated in metastasis in vivo by using a hepatic metastasis model.

\section{Materials and Methods}

\section{Cell Cultures}

Thirteen cell lines derived from human gastric carcinoma were used in this study. Eight gastric cancer cell lines of the HSC series (HSC-57, tubular adenocarcinoma; HSC-42, HSC-58 and HSC-59, poorly differentiated adenocarcinoma; HSC-40, HSC44, HSC-45 and HSC-60, signet ring cell carcinoma) and SH101P4 (tubular adenocarcinoma) were established by one of the authors (K.Y.) [14, 15]. Three cell lines of the MKN series (MKN-1, adenosquamous cell carcinoma; MKN-7 and MKN-74, tubular adenocarcinoma) were provided by Dr. T. Suzuki (Fukushima Medical University, Fukushima) [16, 17]. The TMK-1 cell line (poorly differentiated adenocarcinoma) was a gift from Dr. W. Yasui (Hiroshima University, Hiroshima) [18]. The cells were maintained in RPMI-1640 (GIBCO Life Technologies, Grand Island, N.Y., USA) supplemented with $1 \mathrm{mM}$ L-glutamine, $10 \%$ fetal bovine serum (FBS; GIBCO Life Technologies) and $12.5 \mu \mathrm{g} / \mathrm{ml}$ gentamycin (Sigma, St. Louis, Mo., USA) under $5 \% \mathrm{CO}_{2}$ in humidified air at $37^{\circ} \mathrm{C}$.

RNA Extraction and Quantitative Reverse

Transcription-Polymerase Chain Reaction

Total RNAs from gastric cancer cell lines $\left(1 \times 10^{6}\right)$ were isolated using an RNeasy Mini Kit (Qiagen, Hilden, Germany) ac- cording to the manufacturer's instructions. Quantitative reverse transcription-polymerase chain reaction (RT-PCR) was performed with a SYBR Green real-time quantitative RT-PCR assay kit (Qiagen) on RNA extracts obtained from gastric cancer cell lines using an ABI PRISM 7700 Sequence Detection System (ABI, Carlsbad, Calif., USA). The primer set used for RT-PCR amplification of the PRL-3 was as follows: forward, $5^{\prime}$-GGG ACT TCT CAG GTC GTG TC-3'; reverse, 5'-AGC CCC GTA CTT CTT CAG GT- $3^{\prime}$. As an internal control, the levels of $\beta$-actin expression were also analyzed (forward, $5^{\prime}$-CCA CGA AAC TAC CTT CAA CTC C-3'; reverse, 5'-TCA TAC TCC TGC TGC TTG CTG ATC C- $\left.3^{\prime}\right)$. A master mix (50 $\left.\mu \mathrm{l}\right)$ of the following reaction components was prepared to the indicated end concentration: $25 \mu \mathrm{l}$ of $2 \times$ QuantiTect SYBR Green RT-PCR Master Mix, 10 ng of total RNA, $1 \mathrm{mM}$ of the primer pair, reverse transcriptase, and $0.5 \mu$ l of QuantiTect RT Mix. They were mixed and amplified for 40 or 45 cycles with the following regimen: reverse transcription at $50^{\circ} \mathrm{C}$ for 30 min; denaturation at $94^{\circ} \mathrm{C}$ for $30 \mathrm{~s}$; annealing at $60^{\circ} \mathrm{C}$ for $30 \mathrm{~s}$; extension at $72^{\circ} \mathrm{C}$ for $1 \mathrm{~min}$.

\section{Plasmid Construction and Transfection}

Human PRL-3 cDNA sequence was obtained from the NCBI website (accession No. NM_032611). The siRNA sequence used for PRL-3 gene silencing was designed based on published data as follows: sense, 5'-GAT CCG TGA CCT ATG ACA AAA CGC TTC AAG AGA GCG TTT TGT CAT AGG TCA CTT TTT GGA AA-3'; antisense, 5'-AGC TTT TCC AAA AAG TGA CCT ATG ACA AAA CGC TCT TTG AAG CGT TTT GTC ATA GGT CAC G-3' [13]. As a negative control, siRNA-targeted green fluorescent protein (GFP) was used based on the manufacturer's instructions as follows: sense, 5'-GAT CCG GTT ATG TAC AGG AAC GCA TTC AAG AGA TGC GTT CCT GTA CAT AAC CTT TTT GGA AA-3'; antisense, 5'-GCC AAT AGA TGT CCT TGC GTA AGT TCT CTA CGC AAG GAC ATG TAT TGG AAA AAC CTT TTC GA-3'. These sequences were cloned between BamHI and HindIII restriction sites in pSilencer 3.1-HI/neo plasmids (Applied Biosystems, Carlsbad, Calif., USA) according to the manufacturer's instructions. Briefly, sense siRNA and antisense siRNA oligonucleotides were mixed with annealing solution and incubated at $90^{\circ} \mathrm{C}$ for $3 \mathrm{~min}$ and $37^{\circ} \mathrm{C}$ for $1 \mathrm{~h}$ to form the hairpin siRNA template. Then, annealed siRNA template inserts were ligated into the $\mathrm{pSi}$ lencer vector using T4 DNA ligase by incubating for $3 \mathrm{~h}$ at room temperature. All constructs were sequenced to confirm that the oligonucleotides were correctly inserted.

Before the transfection procedure, SH101-P4 cells were cultured on 24-well plates in RPMI-1640 with 10\% FBS until 75\% confluence. At first, $1 \mu$ l of Oligofectamine (Invitrogen, Carlsbad, Calif., USA) and $1 \mu \mathrm{g}$ of the pSilencer containing PRL-3 siRNA sequence or GFP siRNA sequence or vector DNA were mixed and diluted to a final volume of $20 \mu \mathrm{l}$ and incubated for $15 \mathrm{~min}$ at room temperature. Then $1-\mu l$ volumes of complexes were added to each 24-well plate and incubated in a $\mathrm{CO}_{2}$ incubator. For stable transfection, the SH101-P4 pSilencer vector-transfected cell lines were selected with $0.5 \mathrm{mg} / \mathrm{ml}$ of G-418 (GIBCO Life Technologies) and cloned by limiting dilution.

Cell Invasion Assay

Cell invasion assay was performed using Transwells $(6.5 \mu \mathrm{m}$ in diameter, polycarbonate membrane, $8-\mu \mathrm{m}$ pore size) coated with extracellular matrix gel obtained from Chemicon (Temecu- 
la, Calif., USA). $1 \times 10^{5}$ cells were placed in the upper chamber with $0.5 \mathrm{ml}$ serum-free medium, whereas the lower chamber (24well plate) was loaded with $1 \mathrm{ml}$ of medium containing $10 \%$ FBS. After $48 \mathrm{~h}$ of incubation at $37^{\circ} \mathrm{C}$ with $5 \% \mathrm{CO}_{2}$, the cells were fixed with $4 \%$ paraformaldehyde and then counterstained with hematoxylin. The cells that had migrated into the lower chamber were observed and counted under a light microscope. For each group, the average of quintuplicate samples and their standard error (SE) were calculated.

\section{Cell Proliferation Assay}

Cell growth and survival of PRL-3 siRNA vector-transfected cells were determined using the Premix WST-1 Cell Proliferation Assay System (Takara Biochemicals, Tokyo, Japan), as described elsewhere [13]. Briefly, $1 \times 10^{5}$ cells $(100 \mu \mathrm{l} /$ well $)$ maintained in phenol-red-free medium for $24 \mathrm{~h}$ were inoculated into 96 -well microtiter plates and cultured at $37^{\circ} \mathrm{C}$ for $48 \mathrm{~h}$. After incubation, 10 $\mu \mathrm{l}$ of Premix WST-1 was added to each microculture well, and the plates were incubated for $30 \mathrm{~min}$ at $37^{\circ} \mathrm{C}$, thereafter absorbance was read at $450 \mathrm{~nm}$ using a microplate reader. Absorbance in the cells with no treatment was considered to be $100 \%$. For each group, the average of quintuplicate samples and their SE were calculated.

\section{Hepatic Metastasis Model}

Subconfluent cultures of gastric cancer cells were harvested and washed 3 times with phosphate-buffered saline. SH101-P4 gastric cancer cells $\left(5 \times 10^{5}\right.$ cells in $0.1 \mathrm{ml}$ of phosphate-buffered saline) were injected into the spleen of three groups of 8 -week-old $\mathrm{BALB} / \mathrm{cA} J$ cl-nu female mice: nontreated group, SH101-P4 $(\mathrm{n}=5)$; vector DNA-transfected group, SH101-P4 ${ }^{\text {vector }}(\mathrm{n}=5) ; P R L-3$ siRNA vector-transfected group, SH101-P4 ${ }^{\Delta \mathrm{PRL}-3}(\mathrm{n}=5)$. After 28 days, mice were sacrificed under anesthesia and the liver and the spleen were taken, fixed with $10 \%$ buffered formalin ( $\mathrm{pH} 7.4$ ), then processed for histological examination. Tumor growth was monitored by measuring the number of metastatic foci in 6 sections per liver.

\section{Statistical Analysis}

Student's two-sided t test was used to compare values of test and control samples; $\mathrm{p}$ values less than 0.05 were considered statistically significant. All statistical analyses were performed using StatView software (SAS Institute Inc., Cary, N.C., USA).

\section{Results}

We first examined the mRNA expression levels of $P R L-3$ in 13 gastric cancer cell lines using quantitative RT-PCR (fig. 1). Among 13 gastric cancer cell lines that expressed PRL-3 mRNA at various levels, SH101-P4 showed the highest level of $P R L-3$ mRNA expression while MKN-7 cells revealed the lowest. There were several reports showing correlations between overexpression of PRL-3 and cancer metastasis in several cancers [7, 8, 13]. Therefore, we chose SH101-P4 for the following RNA interference studies as a parental cell.

PRL-3 in Human Gastric Cancer

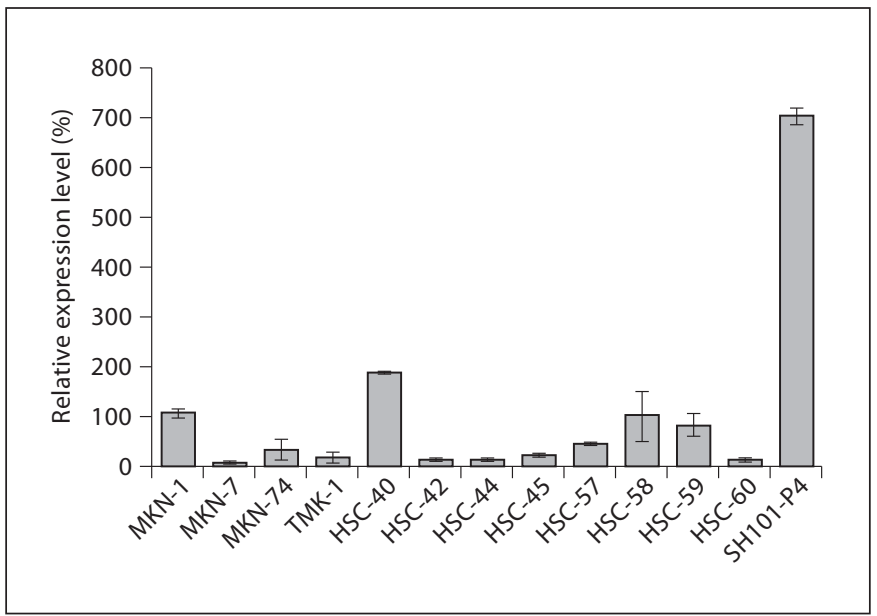

Fig. 1. $P R L-3$ mRNA expression in various human gastric cancer cell lines detected by RT-PCR analysis. The levels of $\beta$-actin mRNA expression were used as an internal control. Relative mRNA expression of each cell line was calculated when the expression of MKN-1 was considered as $100 \%$. The mean value of triplicate samples was plotted with bars indicating SE.

In order to assess the phenotypes of tumor cells in which PRL-3 expression was constitutively suppressed, we established two PRL-3-down-regulated clones (SH101P4 $4^{\triangle \mathrm{PRL}-3-1}$ and SH101-P4 ${ }^{\triangle \mathrm{PRL}-3-2}$ ) and clonal controls (SH101-P4 ${ }^{\text {vector }}$ and SH101-P4 $\left.{ }^{\mathrm{AGFP}}\right)$. The PRL-3 mRNA expression levels in SH101-P4 ${ }^{\triangle \mathrm{PRL}-3-1}$ and SH101-P4 ${ }^{\triangle \mathrm{PRL}-3-2}$ were reduced by 95 and 50\%, respectively, compared with parental SH101-P4. No difference among the parental SH101-P4, SH101-P4 ${ }^{\text {vector }}$ and SH101-P4 ${ }^{\Delta \mathrm{GFP}}$ cells was observed in $P R L-3$ mRNA expression levels (fig. 2a). This RNA interference-mediated effect was specific, as $\beta$-actin levels did not differ significantly among the treated cells and controls. Subclone SH101-P4 ${ }^{\Delta \text { PRL-3-1 }}$ was used in the following experiments.

To evaluate the constitutive knockdown effects of PRL-3 expression on gastric cancer cell invasion in vitro, we performed a Transwell invasion assay. As shown in figure $2 \mathrm{~b}$, the average percentages of cells having migrated were as follows: SH101-P4, $100 \pm 12.0 \%$; SH101P4 ${ }^{\Delta \text { PRL-3-1 }}, 23.9 \pm 6.5 \%$; SH101-P4 ${ }^{\text {vector }}, 95.0 \pm 13.0 \%$; and SH101-P4 ${ }^{\mathrm{AGFP}} 98.5 \pm 11.1 \%$. Cell invasion in vitro was significantly suppressed in SH101-P4 ${ }^{\triangle \mathrm{PRL}-3-1}$ in comparison with SH101-P4 ( $\mathrm{p}=0.0024)$.

Next, we attempted to analyze the effect of PRL-3 knockdown on gastric cancer cell growth in vitro. The average number of cells of each cell line after $50 \mathrm{~h}$ of cultivation, supposing the cell number at the beginning as

Pathobiology 2010;77:155-162 
$100 \%$, was $228 \pm 29.3 \%, 216 \pm 24.4 \%, 219 \pm 19.5 \%$ and $144 \pm 23.5 \%$ in SH101-P4, SH101-P4 ${ }^{\text {vector }}$, SH101-P4 ${ }^{\Delta \mathrm{GFP}}$ and SH101-P4 ${ }^{\triangle \mathrm{PRL}-3-1}$, respectively (fig. $2 \mathrm{c}$ ). $\mathrm{SH} 101-\mathrm{P} 4^{\text {vector }}$ and SH101-P4 ${ }^{\triangle \mathrm{GFP}}$ showed similar cell proliferation activity compared with the parental SH101-P4 cells as determined by WST-1 assay, while SH101-P4 ${ }^{\triangle \mathrm{PRL}-3-1}$ showed significantly suppressed cell proliferation compared with SH101-P4 ( $\mathrm{p}=0.0092)$.

Finally, we evaluated the constitutive PRL-3 knockdown effect on metastasis in vivo using a mouse hepatic metastasis model. SH101-P4 and its subclones were injected into the spleen of BALB/cA mice and the numbers of macroscopic metastatic foci in the liver were counted 4 weeks after tumor inoculation. The mean number of liver metastases for each case was $3.1 \pm 2.9$ and 2.6 \pm 2.4 in SH101-P4 ${ }^{\text {vector }}$ and SH101-P4, respectively. In contrast, the number of metastatic foci markedly decreased in SH101-P4 ${ }^{\Delta \text { PRL-3-1 }}(0.7 \pm 0.7)$. Statistically significant differences in the number of liver metastases were detected between SH101-P4 ${ }^{\Delta \mathrm{PRL}-3-1}$-injected and SH101-P4 ${ }^{\text {vector }}$-injected spleens $(\mathrm{p}=0.0211)$, or between SH101-P4 ${ }^{\triangle \mathrm{PRL}-3-1}$-injected and SH101-P4-injected spleens $(\mathrm{p}=0.0312)$, respectively (fig. 3a). The growth of splenic tumors was evaluated by weighing the whole spleen at the time of sacrifice. The weight of the spleen varied in animals, and mean splenic weight was $215 \pm 131,419 \pm 301$

Fig. 2. Effect of $P R L-3$ siRNA on SH101-P4 gastric cancer cells. a Introduction of $P R L-3$ siRNA in gastric cancer cells down-regulates endogenous $P R L-3$. Relative $P R L-3$ mRNA expression levels of SH101-P4 subclones. The results are shown as the ratio between $P R L-3$ and $\beta$-actin. The $P R L-3$ mRNA expression in SH101P4 ${ }^{\triangle \mathrm{PRL}-3-1}$ and SH101-P4. ${ }^{\mathrm{PRL}-3-2}$ was reduced by 95 and $50 \%$, respectively, compared with parental SH101-P4. The mean value of triplicate samples was plotted with bars indicating SE. b PRL-3 knockdown inhibits gastric cancer cell invasion in vitro. The number of cells invaded from the upper chamber to the lower chamber was counted after $48 \mathrm{~h}$ of incubation. Parental SH101-P4 cell number was considered as $100 \%$ and the ratio of subclonal cells was calculated. Statistically significant differences between SH101-P4 and SH101-P4 ${ }^{\Delta R L-3-1}$, SH101-P4 and SH101-P4 ${ }^{\text {vector }}$, or SH101-P4 and SH101-P4 ${ }^{\Delta \mathrm{GFP}}$ were determined by $\mathrm{t}$ test $\left({ }^{*} \mathrm{p}=\right.$ $\left.0.0024,{ }^{* *} \mathrm{p}=0.7590,{ }^{* *} \mathrm{p}=0.9312\right)$. c Growth of SH101-P4 cell and its subclones. Cell growth was evaluated by WST-1 assay after $48 \mathrm{~h}$ of incubation as described in 'Materials and Methods'. Statistically significant differences between SH101-P4 and SH101P4 ${ }^{\triangle \mathrm{GFP}}, \mathrm{SH} 101-\mathrm{P} 4$ and SH101-P4 ${ }^{\text {vector }}$, or SH101-P4 and SH101$\mathrm{P} 4^{\triangle \mathrm{PRL}-3-1}$ after $50 \mathrm{~h}$ of incubation were determined by $\mathrm{t}$ test $\left({ }^{*} \mathrm{p}=0.7361,{ }^{* *} \mathrm{p}=0.0894,{ }^{* * *} \mathrm{p}=0.0092\right)$. Mean values of quintuplicate samples were plotted with bars indicating SE.
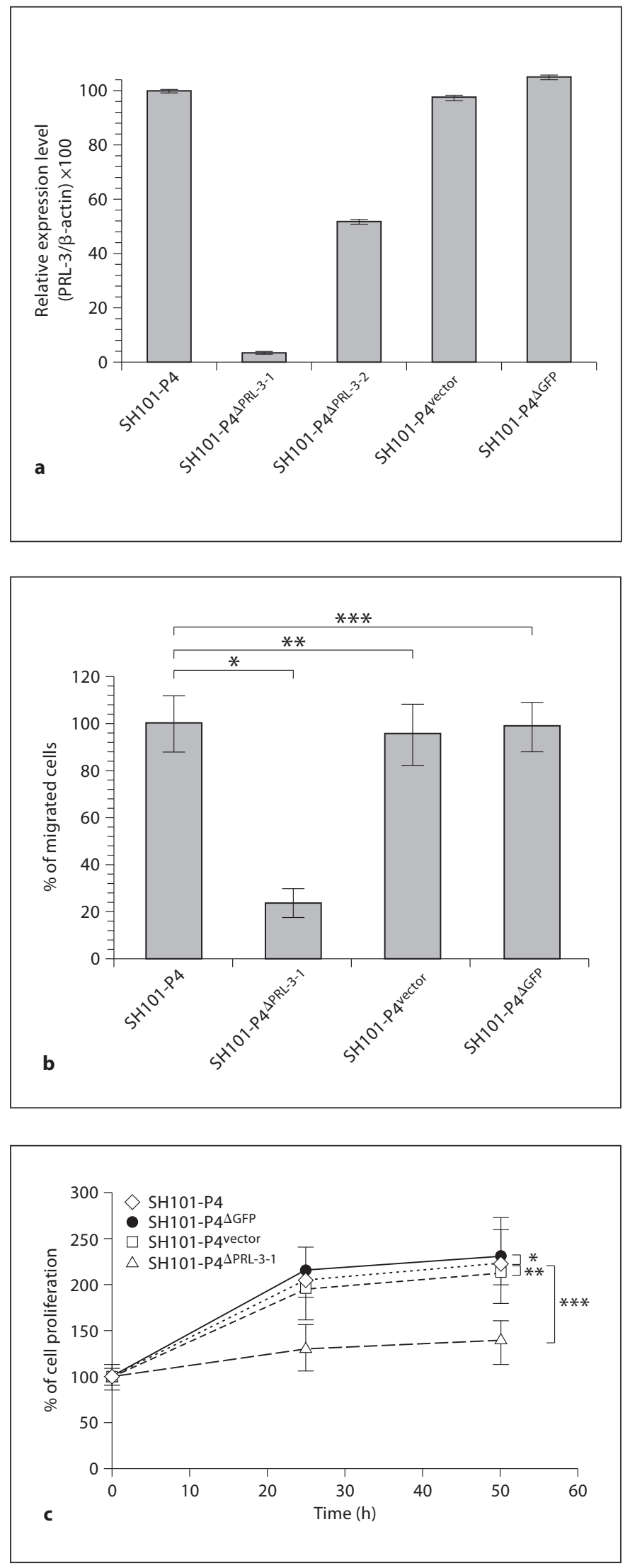

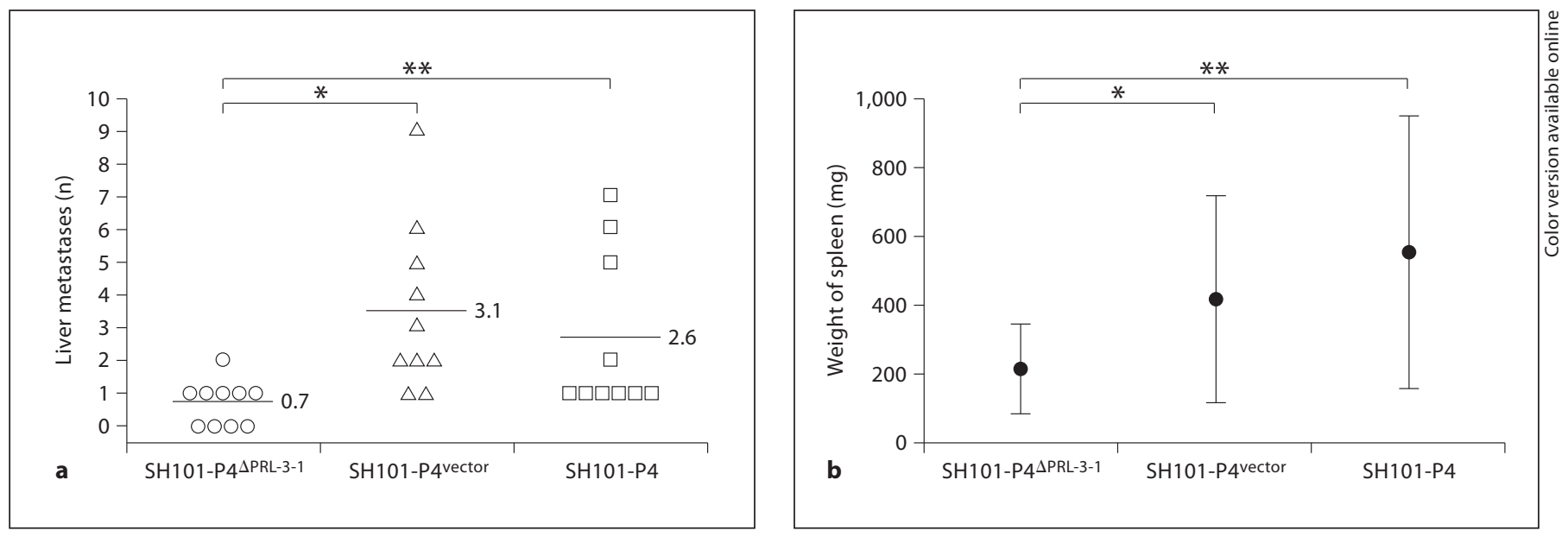

Fig. 3. PRL-3 knockdown resulted in the inhibition of liver metastasis in vivo. SH101-P4 cells and its subclones were injected into the spleen of BALB/cA mice. Mice were sacrificed 28 days after the injection. a Metastatic tumor growth was evaluated by counting the number of metastatic foci in 6 sections per liver. Statistically significant differences between SH101-P4 ${ }^{\triangle \text { PRL-3-1 }}$ and SH101-P4 $4^{\text {vector }}$, or SH101-P4 ${ }^{\triangle \mathrm{PRL}-3-1}$ and SH101-P4 were determined by $\mathrm{t}$ test ${ }^{*} \mathrm{p}=0.0211,{ }^{* *} \mathrm{p}=$ $0.0312)$. Mean values of quintuplicate samples were plotted with error bars. b Primary tumor growth was evaluated by weighing the spleen. Statistically significant differences between SH101-P4 ${ }^{\triangle \mathrm{PRL}-3-1}$ and SH101-P4 ${ }^{\text {vector }}$, or SH101-P4 ${ }^{\Delta \text { PRL-3-1 }}$ and SH101-P4 were determined by $\mathrm{t}$ test $\left({ }^{*} \mathrm{p}=\right.$ $\left.0.257,{ }^{* *} \mathrm{p}=0.391\right)$. c Macroscopic images of representative primary splenic tumor and metastatic hepatic foci. Arrowheads and white arrows indicate the primary splenic tumor and metastatic hepatic foci occurring in a SH101-P4 cell-injected mouse. d Microscopic sections of splenic tumors. HE. $\times 20$. Higher magnification $(\times 200)$ provided in the inset.
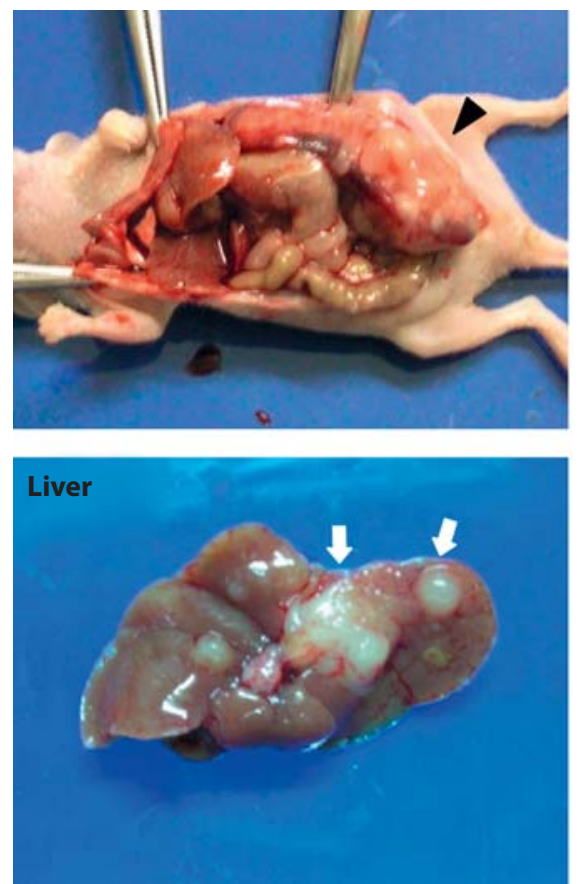

SH101-P4

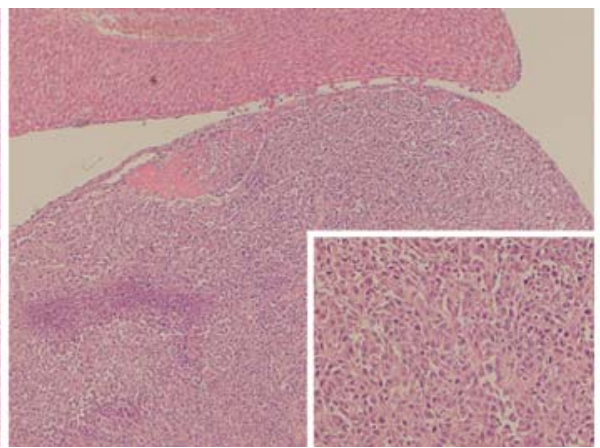

SH101-P4vector
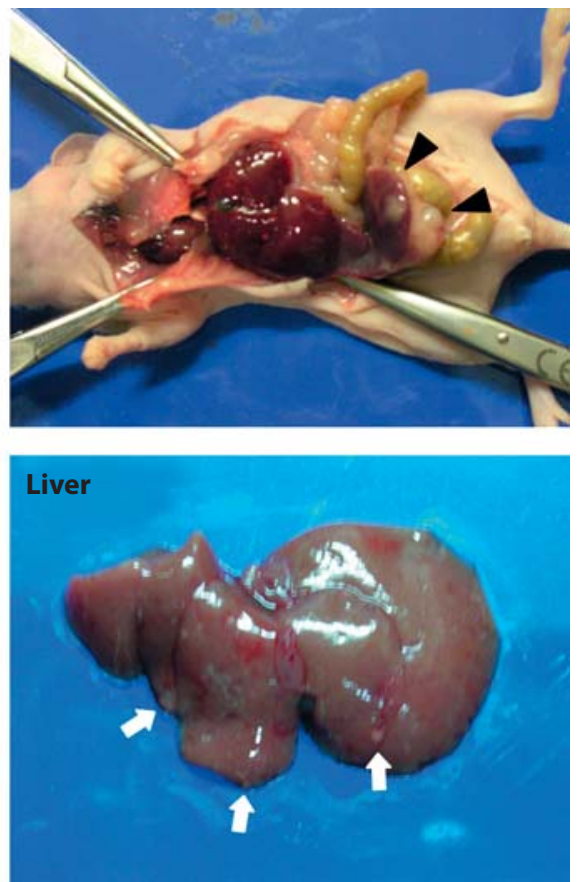

SH101-P4APRL-3-1

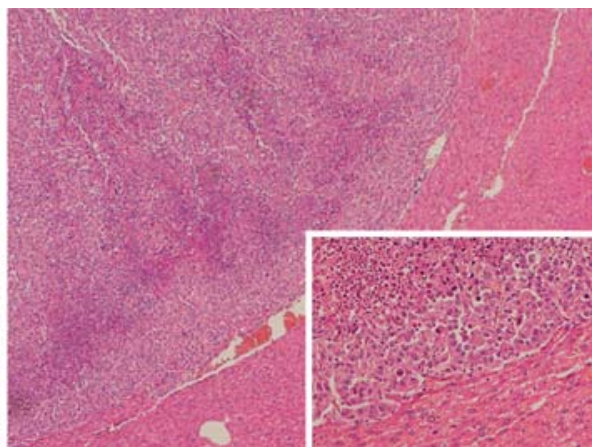

SH101-P4 $\triangle P R L-3-1$ 
and $559 \pm 395 \mathrm{mg}$ in $\mathrm{SH} 101-\mathrm{P} 4^{\Delta \mathrm{PRL}-3-1}, \mathrm{SH} 101-\mathrm{P} 4^{\text {vector }}$ and SH101-P4, respectively (fig. 3b). Although the weights of SH101-P4 ${ }^{\triangle \mathrm{PRL}-3-1}$-injected spleens had a tendency to be smaller than that of controls, no statistical difference was observed (between SH101-P4 ${ }^{\Delta \mathrm{PRL}-3-1}$ and SH101-P4 ${ }^{\text {vector }}$, $\mathrm{p}=0.257$; SH101-P4 ${ }^{\Delta \mathrm{PRL}-3-1}$ and SH101-P4, $\mathrm{p}=0.391$ ).

As shown in figure $3 c$, each cancer cell line formed white nodules clearly demarcated from normal tissue in the injected spleen as well as in the metastatic liver. In addition, 1 out of 10 SH101-P4- and 2 out of 10 SH101$\mathrm{P} 4{ }^{\text {vector }}$-injected mice showed peritoneal dissemination. On the other hand, peritoneal dissemination was not observed in SH101-P4 ${ }^{\triangle \mathrm{PRL}-3-1}$-injected mice. As shown in figure $3 \mathrm{~d}$, the histology of cancer tissues in primary foci was not distinctively different in PRL-3 knockdown and parental cells.

\section{Discussion}

In this study, we examined $P R L-3$ mRNA expression levels in 13 gastric cancer cell lines originating from various histological types. We could not find a correlation between the histological types of these cell lines and the expression levels of $P R L-3$. The expression of $P R L-3$ in SH101-P4 was distinctively higher than that of other cells. Although the reason why SH101-P4 showed high PRL-3 levels remains uncertain, one report showed that only SH101-P4 cells were able to grow in medium without polypeptide growth factor while other HSC-39, HSC-40, HSC-41, HSC-42 and HSC-45 cells required growth factors to be maintained in culture [19]. PRL-3, when highly expressed, might act as a trigger of another cell proliferation pathway, which is independent of known growth factor pathways.

In order to evaluate the effect of PRL-3 on invasion and growth of gastric cancer, we established the SH101P4 ${ }^{\Delta \text { PRL-3-1 }}$ cell in which PRL-3 expression was constantly and semipermanently suppressed by a plasmid vector expressing $P R L$-3-specific siRNA whose sequence was the same as in our previous report [13]. PRL-3 knockdown greatly reduced gastric cancer cell invasion in vitro. Because the growth ability was also reduced greatly, results of cell invasion might be influenced. Cell growth of SH101-P4 ${ }^{\mathrm{DRL}-3-1}$ was reduced by $34 \%$ compared with SH101-P4 after $48 \mathrm{~h}$, while cell invasion of SH101P4 ${ }^{\Delta \text { PRL-3-1 }}$ was reduced by $76 \%$ compared with SH101-P4 after $48 \mathrm{~h}$. Since the reduction rate of cell invasion was much higher than that of cell proliferation by PRL-3 knockdown, we concluded that PRL-3 plays an important role in cell invasion ability. Similar results were obtained in our previous study with transient transfection of oligonucleotide siRNA against PRL-3 to DLD-1 colon cancer cells [13]. The effect of PRL-3 on cell motility has been reported not only in gastric and colon cancer, but also in some other types of cancer cells such as human myeloma cells and mouse melanoma cells [20,21]. Although the mechanism of how PRL-3 works upon cell invasion is still undefined, several reports have been published which clarified the PRL-3 target molecules while some of them are known to be involved in cell motility [22]. For example, Peng et al. [22] reported that integrin $\alpha 1$ directly interacted with PRL-3 using a yeast two-hybrid method. They also reported that PRL-3 directly down-regulated the phosphorylation level of integrin $\beta 1$ and up-regulated the phosphorylation level of Erk1/2, which are involved in cell motility [22]. Wang et al. [23] showed that PRL-3 promoted epithelial-mesenchymal transition, leading to metastatic cancer by down-regulating PTEN via PI3K pathways. Recently, Mizuuchi et al. [24] reported the direct involvement of PRL-3 in cell invasion by isolating a molecule, intermediate filament keratin 8 (KRT8), which was directly dephosphorylated by PRL-3. Therefore, PRL-3 may regulate cell invasion by modulation of phosphorylation state of known or unknown molecular targets.

On the other hand, the effect of PRL-3 on cell growth is somewhat controversial. In this study, contribution of PRL-3 not only to cell motility, but also to cell growth in vitro and in vivo was suggested. Recently, Cai et al. [25] and Li et al. [26] reported that gastric cancer cell proliferation and motility were suppressed when PRL-3 expression was inhibited by microRNA system. Our result was consistent with these reports. Certainly, cell proliferation and motility are derived from totally different pathways, however, there are some molecules which work on both cell growth and motility. For example, PRL-1, a member of the tyrosine phosphatase family, was reported to be involved in both cell growth and motility $[4,6]$. Overexpression of PRL-1 led to high cellular growth while PRL-1 was located primarily in the nucleus [4]. PRL-1 was firstly reported to be localized in the nucleus, but Wang et al. [27] demonstrated that PRL-1 moves from the endoplasmic reticulum during the interphase to the centrosomes in the mitotic phase. PRL-1 in the interphase acted in a prenylation-dependent manner while PRL-1 in the mitotic phase was expected to work on cell division by regulating its spindle dynamics in a prenylation-independent manner [27]. In addition, PRL-1 promotes cell motility in a prenylation-dependent manner 
[28]. Since more than $75 \%$ of amino acid sequences of PRL- 1 and PRL-3 are identical and both contain the Cterminal prenylation motif CAAX to be prenylated $[2$, 29], it is comprehensible that PRL-3 might possess this dual ability, a prenylation-dependent and a prenylationindependent manner like PRL-1.

In this report, liver metastasis was reduced by about $77 \%$ in SH101-P4 ${ }^{\triangle \mathrm{PRL}-3-1}$ cells whose PRL-3 mRNA expression was reduced by $95 \%$ compared with SH101-P4 cells. The percentage of liver metastasis suppression was lower than that of $P R L-3$ mRNA suppression. Percentage discrepancy between $P R L-3$ expression and metastasis formation was reported in another report. Li et al. [26] reported a relationship between $P R L-3$ expression and peritoneal metastasis in gastric cancer using microRNA. In their report, peritoneal dissemination was reduced by about one third, while $P R L-3$ mRNA expression was reduced by more than $80 \%$ [26]. These results may indicate that there might be another molecule which acts to form gastric cancer metastasis. However, PRL-3 may contribute to the establishment of metastasis since the effects of PRL-3 knockdown were experimentally significant.

It is likely to take some time before the exact role of PRL-3 in cell growth and motility can be clarified and surely further study will be needed. However, PRL-3 may be one of the candidates for a molecular target for anticancer therapy by controlling both tumor growth and tumor metastasis.

\section{Acknowledgments}

Supported by Grants-in-Aid for Cancer Research from the Ministry of Health, Labor and Welfare of Japan (14-7 and 20-12). Also supported in part by Grants-in-Aid for Scientific Research (B-14510070, C-19590347, C-20590341) and Exploratory Research (18659096) from the Japan Society for Promotion of Science. A grant from the Terry Fox Run Foundation for Cancer Research was also received.

\section{References}

1 Zhang ZY, Zhou B, Xie L: Modulation of protein kinase signaling by protein phosphatases and inhibitors. Pharmacol Ther 2002;93: 307-317.

-2 Zeng Q, Hong W, Tan YH: Mouse PRL-2 and PRL-3, two potentially prenylated protein tyrosine phosphatases homologous to PRL1. Biochem Biophys Res Commun 1998;244: 421-427.

- 3 Cates CA, Michael RL, Stayrook KR, Harvey KA, Burke YD, Randall SK, Crowell PL, Crowell DN: Prenylation of oncogenic human PTP(CAAX) protein tyrosine phosphatases. Cancer Lett 1996;110:49-55.

- 4 Diamond RH, Cressman DE, Laz TM, Abrams CS, Taub R: PRL-1, a unique nuclear protein tyrosine phosphatase, affects cell growth. Mol Cell Biol 1994;14:3752-3762.

-5 Matter WF, Estridge T, Zhang C, Belagaje R, Stancato L, Dixon J, Johnson B, Bloem L, Pickard T, Donaghue M, Acton S, Jeyaseelan R, Kadambi V, Vlahos CJ: Role of PRL-3, a human muscle-specific tyrosine phosphatase, in angiotensin-II signaling. Biochem Biophys Res Commun 2001;283:1061-1068.

-6 Zeng Q, Dong JM, Guo K, Li J, Tan HX, Koh V, Pallen CJ, Manser E, Hong W: PRL-3 and PRL-1 promote cell migration, invasion, and metastasis. Cancer Res 2003;63:2716-2722.

7 Peng L, Ning J, Meng L, Shou C: The association of the expression level of protein tyrosine phosphatase PRL-3 protein with liver metastasis and prognosis of patients with colorectal cancer. J Cancer Res Clin Oncol 2004;130:521-526.
-8 Wu X, Zeng H, Zhang X, Zhao Y, Sha H, Ge X, Zhang M, Gao X, Xu Q: Phosphatase of regenerating liver-3 promotes motility and metastasis of mouse melanoma cells. Am J Pathol 2004;164:2039-2054.

-9 Radke I, Götte M, Kersting C, Mattsson B, Kiesel L, Wülfing P: Expression and prognostic impact of the protein tyrosine phosphatases PRL-1, PRL-2, and PRL-3 in breast cancer. Br J Cancer 2006;95:347-354

-10 Stephens B, Han H, Hostetter G, Demeure MJ, Von Hoff DD: Small interfering RNAmediated knockdown of PRL phosphatases results in altered Akt phosphorylation and reduced clonogenicity of pancreatic cancer cells. Mol Cancer Ther 2008;7:202-210.

-11 Zhou J, Wang S, Lu J, Li J, Ding Y: Over-expression of phosphatase of regenerating liver-3 correlates with tumor progression and poor prognosis in nasopharyngeal carcinoma. Int J Cancer 2009;124:1879-1886.

12 Miskad UA, Semba S, Kato H, Matsukawa Y, Kodama Y, Mizuuchi E, Maeda N, Yanagihara K, Yokozaki H: High PRL-3 expression in human gastric cancer is a marker of metastasis and grades of malignancies: an in situ hybridization study. Virchows Arch 2007;450:303-310.

13 Kato H, Semba S, Miskad UA, Seo Y, Kasuga M, Yokozaki H: High expression of PRL-3 promotes cancer cell motility and liver metastasis in human colorectal cancer: a predictive molecular marker of metachronous liver and lung metastases. Clin Cancer Res 2004; 10:7318-7328.
14 Yanagihara K, Ito A, Toge T, Numoto M: Antiproliferative effects of isoflavones on human cancer cell lines established from the gastrointestinal tract. Cancer Res 1993;53: 5815-5821.

15 Yanagihara K, Seyama T, Tsumuraya M, Kamada N, Yokoro K: Establishment and characterization of human signet ring cell gastric carcinoma cell lines with amplification of the c-myc oncogene. Cancer Res 1991;51: 381-386.

16 Hojo H: Establishment of cultured cell lines of human stomach cancer - origin and their morphological characteristics (in Japanese). Niigata Igakukai Zasshi 1977;91:737-752.

17 Motoyama T, Hojo H, Watanabe H: Comparison of seven cell lines derived from human gastric carcinomas. Acta Pathol Jpn 1986;36:65-83.

18 Ochiai A, Yasui W, Tahara E: Growth-promoting effect of gastrin on human gastric carcinoma cell line TMK-1. Jpn J Cancer Res 1985;76:1064-1071

19 Yanagihara K, Ito A, Toge T, Numoto M: Antiproliferative effects of isoflavones on human cancer cell lines established from the gastrointestinal tract. Cancer Res 1993;53: 5815-5821.

20 Fagerli UM, Holt RU, Holien T, Vaatsveen TK, Zhan F, Egeberg KW, Barlogie B, Waage A, Aarset H, Dai HY, Shaughnessy JD Jr, Sundan A, Børset M: Overexpression and involvement in migration by the metastasisassociated phosphatase PRL-3 in human myeloma cells. Blood 2008;111:806-815. 
-21 Qian F, Li YP, Sheng X, Zhang ZC, Song R, Dong W, Cao SX, Hua ZC, Xu Q: PRL-3 siRNA inhibits the metastasis of B16-BL6 mouse melanoma cells in vitro and in vivo. Mol Med 2007;13:151-159.

-22 Peng L, Jin G, Wang L, Guo J, Meng L, Shou C: Identification of integrin alphal as an interacting protein of protein tyrosine phosphatase PRL-3. Biochem Biophys Res Commun 2006;342:179-183.

-23 Wang H, Quah SY, Dong JM, Manser E, Tang JP, Zeng Q: PRL-3 down-regulates PTEN expression and signals through PI3K to promote epithelial-mesenchymal transition. Cancer Res 2007;67:2922-2926.
24 Mizuuchi E, Semba S, Kodama Y, Yokozaki $\mathrm{H}$ : Down-modulation of keratin 8 phosphorylation levels by PRL-3 contributes to colorectal carcinoma progression. Int J Cancer 2009;124:1802-1810.

25 Cai SR, Wang Z, Chen CQ, Wu WH, He YL, Zhan WH, Zhang CH, Cui J, Wu H: Role of silencing phosphatase of regeneration liver-3 expression by microRNA interference in the growth of gastric cancer. Chin Med J (Engl) 2008;121:2534-2538.

26 Li Z, Zhan W, Wang Z, Zhu B, He Y, Peng J, Cai S, Ma J: Inhibition of PRL-3 gene expression in gastric cancer cell line SGC7901 via microRNA suppressed reduces peritoneal metastasis. Biochem Biophys Res Commun 2006;348:229-237.
27 Wang J, Kirby CE, Herbst R: The tyrosine phosphatase PRL-1 localizes to the endoplasmic reticulum and the mitotic spindle and is required for normal mitosis. J Biol Chem 2002;277:46659-46668.

28 Fiordalisi JJ, Keller PJ, Cox AD: PRL tyrosine phosphatases regulate rho family GTPases to promote invasion and motility. Cancer Res 2006;66:3153-3161.

29 Cates CA, Michael RL, Stayrook KR, Harvey KA, Burke YD, Randall SK, Crowell PL, Crowell DN: Prenylation of oncogenic human PTP(CAAX) protein tyrosine phosphatases. Cancer Lett 1996;110:49-55. 